\title{
Generalized Discrete Finite Fourier Series with Two Parameter
}

Govindan $\mathrm{B}^{*}[1]$ and Raji $\mathrm{N}^{2}$

Received: 28 March 2021/ Accepted: 15 June 2021/ Published online: 18 June 2021

(C)Sacred Heart Research Publications 2017

\begin{abstract}
In this paper, we obtain a Generalized Discrete Finite Fourier Series by using generalized difference operator with two parameters. Suitable examples are given to illustrate the results.

Key words: Difference Equation, Generalized Difference Operator, $\ell$-Difference Operator, Fourier Series, Polynomial.
\end{abstract}

AMS classification: 39A70, 39A10, 47B39, 80A20.

\section{Introduction}

The Fourier Series is the most widely used series expansion in mathematics modeling of engineering systems. It serves as the basis for the Fourier integral, the Laplace transform, the solution of autonomous linear differential equations, frequency response methods and many engineering applications. There are many good treatments on the subject; too many to mention in a comprehensive manner. However, the treatment by Tolstov[9] is classical. Jerri[5] provides an excellent overview on convergence of the Fourier series and discusses Gibbs-like phenomena in continuous and discrete wavelet representations. Sidi[7] reviews the state of the art of extrapolation methods giving applied scientists and engineers a practical guide to accelerating convergence in difficult computational problems. Also, accelerated convergence by means of periodic bridge functions was developed by Anguelov[1].

A Fourier Series is a series of sines and cosines of an angle and its multiples of the form $\frac{a_{0}}{2}+a_{1} \cos k+a_{2} \cos 2 k+a_{3} \cos 3 k+\ldots+a_{\omega} \cos \omega k+\ldots+b_{1} \sin k+$ $b_{2} \sin 2 k+b_{3} \sin 3 k \ldots+b_{\omega} \sin \omega k \ldots=\frac{a_{0}}{2}+\sum_{\omega=1}^{\infty} a_{\omega} \cos \omega k+\sum_{\omega=1}^{\infty} b_{\omega} \sin \omega k$. is called the

\footnotetext{
${ }^{1}$ Department of Mathematics, Sacred Heart College, Tirupattur - 635 601, Tamil Nadu, S.India.

${ }^{2}$ Department of Mathematics, KMG College of Arts and Science, Gudiyattam - 632 602, Tamil Nadu, S.India.

Email: ${ }^{*, 1}$ govind.viveka@gmail.com, ${ }^{2}$ way2raji@@gmail.com
} 
Fourier series. Where $a_{1}, a_{2}, \ldots, a_{\omega}, \ldots b_{1}, b_{2}, \ldots b_{\omega}, \ldots$ are constants.

A periodic function $f(x)$ can be expanded in a Fourier Series. The series consists of the following: (i) A constant term $a_{0}$. (ii) A component at the fundamental frequency determined by the values of $a_{1}, b_{1}$. (iii) Components of the harmonics (multiples of the fundamental frequency) determined by $a_{2}, a_{3}, \ldots b_{2}, b_{3}, \ldots$ and $a_{0}, a_{1}, a_{2}, \ldots b_{1}, b_{2}, \ldots$ are known as Fourier coefficients of Fourier constants. And the Fourier coefficients are defined by,

$a_{0}=\frac{1}{\pi} \int_{0}^{2 \pi} f(x) d x, a_{\omega}=\frac{1}{\pi} \int_{0}^{2 \pi} f(x) \cos \omega x d x$ and $b_{\omega}=\frac{1}{\pi} \int_{0}^{2 \pi} f(x) \sin \omega x d x$.

\section{Discrete Fourier series}

The discrete Fourier series may be studied merely from a mathematical point of view. But, it is also of interest to know where it originated or where it is applied. As far as the author is aware, it originated in telecommunication engineering in connection with the digitalization of data (signals) in order to transmit them. Formerly, signals were transmitted in the form of waves that were a superposition of continuous periodic time functions [8]. Nowadays, the signals are digitalized that is to say, they are sampled at discrete points and are then transmitted. In this context, the fast Fourier transform (FFT) allows one to transmit the digitalized (discrete) signals much faster than the pertinent analog (continuous) signals. Thus, there is a very important application of the discrete Fourier series.

Definition 2.1 Let $S_{\ell}=\left\{\phi_{0}, \phi_{1}, \phi_{2}, \ldots \phi_{\eta}\right\}$ be an orthonormal on $I=[a, a+2 \pi], \ell=\frac{\pi}{\eta}, u(k)$ is bounded function on $I$ and $c_{\omega}=\left(u, \phi_{\omega}\right)_{\ell}$, say finite Fourier coefficients. Then the FFS of $u(k)$ related to $S_{\ell}$ is defined as

$$
u(k)=\sum_{\omega=0}^{\eta} c_{\omega} \phi_{\omega}(k), \quad k \in\{a, a+\ell, a+2 \ell, \cdots, a+(\eta-1) \ell\}
$$

\section{Generalized finite Fourier series}

In this section, we present some basic definitions and results.

Definition 3.1 [6] Let $u(k), k \in[0, \infty)$, be a real or complex valued function and 
ISSN: 2456-8686, 5(1), 2021:088-096

https://doi.org/10.26524/cm95

$\ell>0$ be a fixed shift value. Then, the $\ell$-difference operator $\Delta_{\ell}$ on $u(k)$ is defined as

$$
\Delta_{\ell} u(k)=u(k+\ell)-u(k),
$$

and its infinite $\ell$ - difference sum is defined by

$$
\Delta_{\ell}^{-1} u(k)=\sum_{r=0}^{\infty} u(k+r \ell)
$$

Lemma 3.2 [2] Let $p$ be real $\ell>0, k \in(\ell, \infty)$ and $p \ell \neq \beta 2 \pi$. Then we have

$$
\Delta_{\ell}^{-1} \sin p k=\frac{(\sin p(k-\ell)-\sin p k)}{2(1-\cos p \ell)}+c_{j},
$$

and

$$
\Delta_{\ell}^{-1} \operatorname{cosp} k=\frac{(\operatorname{cosp}(k-\ell)-\operatorname{cosp} k)}{2(1-\operatorname{cosp} \ell)}+c_{j}
$$

In particular

$$
\left.\Delta_{\ell}^{-1} \sin p k\right|_{0} ^{2 \pi}=0=\left.\Delta_{\ell}^{-1} \cos p k\right|_{0} ^{2 \pi} .
$$

Definition 3.3 [4] The generalized finite Fourier series is defined by

$$
u(k)=\frac{a_{0}}{2}+\sum_{\omega=1}^{\eta-1}\left(a_{\omega} \cos \omega k+b_{\omega} \sin \omega k\right)+\frac{a_{\eta}}{2} \cos \eta k, \quad k \in\{a+r \ell\}_{r=0}^{2 \eta-1}
$$

where $a_{\omega}=\left.\frac{\ell}{\pi} \Delta_{\ell}^{-1} u(k) \cos \omega k\right|_{a} ^{a+2 \pi}, b_{\omega}=\left.\frac{\ell}{\pi} \Delta_{\ell}^{-1} u(k) \sin \omega k\right|_{a} ^{a+2 \pi}$.

Lemma 3.4 If $k \in(-\infty, \infty)$ and $\ell_{1}, \ell_{2}>0$, then $\Delta_{\ell_{2}}^{-1} \Delta_{\ell_{1}}^{-1} 2^{k}=\frac{2^{k}}{\left(2^{\ell_{1}}-1\right)\left(2^{\ell_{2}}-1\right)}$.

Lemma 3.5 Let $\ell_{1}, \ell_{2}>0$ and $u(k), w(k)$ are real valued bounded functions. Then $\Delta_{\ell_{2}}^{-1} \Delta_{\ell_{1}}^{-1} u(k) v(k)=\Delta_{\ell_{2}}^{-1}\left[u(k) \Delta_{\ell_{1}}^{-1} v(k)\right]-\Delta_{\ell_{2}}^{-1}\left[\Delta_{\ell_{1}}^{-1}\left(\Delta_{\ell_{1}}^{-1} v\left(k+\ell_{1}\right) \Delta_{\ell_{1}} u(k)\right]\right.$.

Theorem 3.6 Let $k \in(-\infty, \infty)$ and $\ell_{1}, \ell_{2}>0$, then we have 
ISSN: 2456-8686, 5(1), 2021:088-096

https://doi.org/10.26524/cm95

$$
\begin{aligned}
& \sum_{r_{1}=0}^{\left[\frac{k}{\ell_{1}}\right]} \sum_{r_{2}=0}^{\left[\frac{k-r_{1} \ell_{1}}{\ell_{2}}\right]}\left(k-r_{2} \ell_{2}-r_{1} \ell_{1}\right) 2^{k-r_{2} \ell_{2}-r_{1} \ell_{1}} \\
& =\left(\frac{\left(k+\ell_{2}+\ell_{1}\right) 2^{k+\ell_{2}+\ell_{1}}}{\left(2^{\ell_{1}}-1\right)\left(2^{\ell_{2}}-1\right)}-\frac{\ell_{2} 2^{\ell_{2}} 2^{k+\ell_{2}+\ell_{1}}}{\left(2^{\ell_{2}}-1\right)^{2}\left(2^{\ell_{1}}-1\right)}-\frac{\ell_{1} 2^{\ell_{1}} 2^{k+\ell_{2}+\ell_{1}}}{\left(2^{\ell_{1}}-1\right)^{2}\left(2^{\ell_{2}}-1\right)}\right) \\
& -\left(\frac{\ell_{2}+\hat{\ell}_{1}(k) 2^{\ell_{2}+\hat{\ell_{1}}(k)}}{\left(2^{\ell_{1}}-1\right)\left(2^{\ell_{2}}-1\right)}-\frac{\ell_{2} 2^{\ell_{2}} 2^{\ell_{2}+\hat{\ell}_{1}(k)}}{\left(2^{\ell_{2}}-1\right)^{2}\left(2^{\ell_{1}}-1\right)}-\frac{\ell_{1} 2^{\ell_{1}} 2^{\ell_{2}+\hat{\ell}_{1}(k)}}{\left(2^{\ell_{1}}-1\right)^{2}\left(2^{\ell_{2}}-1\right)}\right)
\end{aligned}
$$

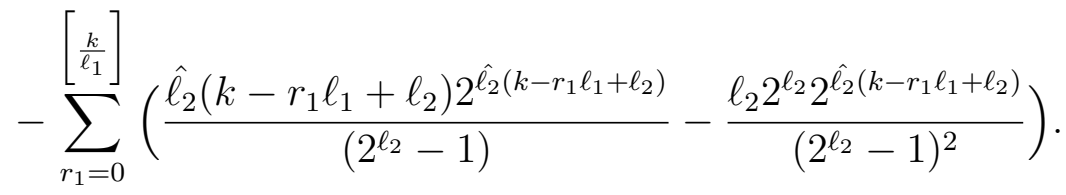

Proof: By using the lemmas (3.4) and (3.5), we get the proof of (8).

Example 3.7 Taking $k=4, \ell_{1}=1$ and $\ell_{2}=2$ in $(3.6)$, we get the summation is equal to 107.5

Theorem 3.8 Let $k \in(-\infty, \infty)$ and $\ell_{1}, \ell_{2}>0$, then we have

$$
\begin{aligned}
& \Delta_{\ell_{2}}^{-1} \Delta_{\ell_{1}}^{-1} k \sin k=\sum_{r_{1}=0}^{\left[\frac{k}{\ell_{1}}\right]} \sum_{r_{2}=0}^{\left.\frac{k-r_{1} \ell_{1}}{\ell_{2}}\right]}\left(k-r_{2} \ell_{2}-r_{1} \ell_{1}\right) \sin \left(k-r_{2} \ell_{2}-r_{1} \ell_{1}\right) \\
&=\frac{1}{2\left(1-\cos \ell_{1}\right)}\left[k \frac{\left(\sin \left(k-\ell_{1}-\ell_{2}\right)-\sin \left(k-\ell_{1}\right)-\sin \left(k-\ell_{2}\right)+\sin k\right)}{2\left(1-\cos \ell_{2}\right)}\right. \\
& \quad-\frac{\ell_{2}}{4\left(1-\cos \ell_{2}\right)^{2}}\left(\sin \left(k-\ell_{1}-\ell_{2}\right)-2 \sin \left(k-\ell_{1}\right)+\sin \left(k-\ell_{1}+\ell_{2}\right)+\sin \left(k-\ell_{2}\right)\right. \\
&\left.\left.2 \sin k+\sin \left(k+\ell_{2}\right)\right)\right]-\frac{\ell_{1}}{4\left(1-\cos \ell_{1}\right)^{2} 2\left(1-\cos \ell_{2}\right)}\left[\sin \left(k-\ell_{1}-\ell_{2}\right)-\sin \left(k-\ell_{1}\right)\right. \\
&\left.-\sin \left(k-\ell_{2}\right)+\sin k-\sin \left(k-\ell_{2}\right)+\sin k+\sin \left(k+\ell_{1}-\ell_{2}\right)-\sin \left(k+\ell_{1}\right)\right] .
\end{aligned}
$$

Proof: Taking $u(k)=k$ and $v(k)=\sin k$ in $(3.5)$, we get the proof of $(9)$.

Corollary 3.9 Let $\ell_{1}, \ell_{2}>0, k \in(-\infty, \infty)$ then we have 
ISSN: 2456-8686, 5(1), 2021:088-096

https://doi.org/10.26524/cm95

$\Delta_{\ell_{2}}^{-1} \Delta_{\ell_{1}}^{-1} \sin p k=\frac{\sin p\left(k-\ell_{2}-\ell_{1}\right)-\sin p\left(k-\ell_{1}\right)-\sin p\left(k-\ell_{2}\right)+\sin p k}{4\left(1-\cos p \ell_{1}\right)\left(1-\cos p \ell_{2}\right)}$

Definition 3.10 The generalized finite Fourier series with two parameter $\left(G F F S_{2}\right)$ is defined by

$$
u(k)=\frac{a_{0}}{2}+\sum_{\omega=1}^{\eta-1}\left(a_{\omega} \cos \omega k+b_{\omega} \sin \omega k\right)+\frac{a_{\eta}}{2} \cos \eta k, \quad k \in\{a+r \ell\}_{r=0}^{2 \eta-1},
$$

$a_{\omega}=\left.\left.\frac{\ell_{1}+\ell_{2}}{\pi} \Delta_{\ell_{2}}^{-1} \Delta_{\ell_{1}}^{-1} u(k) \cos \omega k\right|_{a} ^{a+2 \pi}\right|_{a} ^{a+2 \pi}, b_{\omega}=\left.\left.\frac{\ell_{1}+\ell_{2}}{\pi} \Delta_{\ell_{2}}^{-1} \Delta_{\ell_{1}}^{-1} u(k) \sin \omega k\right|_{a} ^{a+2 \pi}\right|_{a} ^{a+2 \pi}$.

Theorem 3.11 Let $\ell_{1}$ and $\ell_{2}>0$ and $k \in(-\infty, \infty)$, then $G F F S_{2}$ of $k 2^{k}$ is

$$
\begin{aligned}
& k 2^{k}=\frac{\ell_{1}+\ell_{2}}{\pi}\left\{\left(\frac{\left(k+\ell_{2}+\ell_{1}\right) 2^{k+\ell_{2}+\ell_{1}}}{\left(2^{\ell_{1}}-1\right)\left(2^{\ell_{2}}-1\right)}-\frac{\ell_{2} 2^{\ell_{2}} 2^{k+\ell_{2}+\ell_{1}}}{\left(2^{\ell_{2}}-1\right)^{2}\left(2^{\ell_{1}}-1\right)}-\frac{\ell_{1} 2^{\ell_{1}} 2^{k+\ell_{2}+\ell_{1}}}{\left(2^{\ell_{1}}-1\right)^{2}\left(2^{\ell_{2}}-1\right)}\right)\right. \\
& -\left(\frac{\ell_{2}+\hat{\ell_{1}}(k) 2^{\ell_{2}+\hat{\ell_{1}}(k)}}{\left(2^{\ell_{1}}-1\right)\left(2^{\ell_{2}}-1\right)}-\frac{\ell_{2} 2^{\ell_{2}} 2^{\ell_{2}+\hat{\ell}_{1}(k)}}{\left(2^{\ell_{2}}-1\right)^{2}\left(2^{\ell_{1}}-1\right)}-\frac{\ell_{1} 2^{\ell_{1}} 2^{\ell_{2}+\hat{\ell}_{1}(k)}}{\left(2^{\ell_{1}}-1\right)^{2}\left(2^{\ell_{2}}-1\right)}\right) \\
& -\sum_{r_{1}=0}^{\left[\frac{k}{\ell_{1}}\right]}\left(\frac{\hat{\ell_{2}}\left(k-r_{1} \ell_{1}+\ell_{2}\right) 2^{\hat{\ell}_{2}\left(k-r_{1} \ell_{1}+\ell_{2}\right)}}{\left(2^{\ell_{2}}-1\right)}-\frac{\ell_{2} 2^{\ell_{2}} 2^{\ell_{\ell_{2}}\left(k-r_{1} \ell_{1}+\ell_{2}\right)}}{\left(2^{\ell_{2}}-1\right)^{2}}\right)+\sum_{\omega=1}^{\eta-1} \sum_{r_{1}=0}^{\left[\frac{k}{\ell_{1}}\right]} \sum_{r_{2}=0}^{\left[\frac{k-r_{1} \ell_{1}}{\ell_{2}}\right]} \\
& \left.\left(\mu(k) \sin \omega\left(k-r_{2} \ell_{2}-r_{1} \ell_{1}\right) \sin \omega k+\mu(k) \cos \omega\left(k-r_{2} \ell_{2}-r_{1} \ell_{1}\right) \cos \omega k\right)\right\} .
\end{aligned}
$$

Where $\mu(k)=\left(k-r_{2} \ell_{2}-r_{1} \ell_{1}\right) 2^{\left(k-r_{2} \ell_{2}-r_{1} \ell_{1}\right)}$

Proof: The proof of (11) obtained by using (10) and (9).

\section{Odd and Even function}

Definition 4.1 (Odd and Even function) Even functions are the functions for which the left half of the plane looks like the mirror image of the right of the plane. Odd functions are the functions where the left half of the plane looks like the mirror image of the right of the plane,only upside-down.

The function $u(k)$ is an even function if $u(-k)=u(k)$ and odd if $u(-k)=-u(k)$.

Definition 4.2 The generalized finite half-range Fourier series $\left(G F F S_{2}\right)$ of odd and 
ISSN: 2456-8686, 5(1), 2021:088-096

https://doi.org/10.26524/cm95

even functions are is defined as If $u(k)$ is even function, then 10 becomes,

$$
u(k)=\frac{a_{0}}{2}+\sum_{\omega=1}^{\eta-1} a_{\omega} \cos \omega k+\frac{a_{\eta}}{2} \cos \eta k
$$

where $a_{\omega}=\left.\left.\frac{\ell_{1}+\ell_{2}}{\pi} \Delta_{\ell_{2}}^{-1} \Delta_{\ell_{1}}^{-1} u(k) \cos \omega k\right|_{a} ^{a+2 \pi}\right|_{a} ^{a+2 \pi}$.

If $u(k)$ is odd function, then $(10)$ becomes,

$$
u(k)=\sum_{\omega=1}^{\eta-1} b_{\omega} \sin \omega k,
$$

where $b_{\omega}=\left.\left.\frac{\ell_{1}+\ell_{2}}{\pi} \Delta_{\ell_{2}}^{-1} \Delta_{\ell_{1}}^{-1} u(k) \sin \omega k\right|_{a} ^{a+2 \pi}\right|_{a} ^{a+2 \pi}$.

Theorem 4.3 Let $u(k)=k, \ell_{1}$ and $\ell_{2}>0$, then $G F F S_{2}$ of $k$ is

$$
\begin{aligned}
k= & \frac{\ell_{1}+\ell_{2}}{\pi} \sum_{\omega=1}^{\eta-1} \frac{1}{2\left(1-\cos \ell_{1}\right)}\left[k \frac{\left(\sin \left(k-\ell_{1}-\ell_{2}\right)-\sin \left(k-\ell_{1}\right)-\sin \left(k-\ell_{2}\right)+\sin k\right)}{2\left(1-\cos \ell_{2}\right)}\right. \\
& -\frac{\ell_{2}}{4\left(1-\cos \ell_{2}\right)^{2}}\left(\sin \left(k-\ell_{1}-\ell_{2}\right)-2 \sin \left(k-\ell_{1}\right)+\sin \left(k-\ell_{1}+\ell_{2}\right)+\sin \left(k-\ell_{2}\right)\right. \\
& \left.\left.-2 \sin k+\sin \left(k+\ell_{2}\right)\right)\right]-\frac{\ell_{1}}{4\left(1-\cos \ell_{1}\right)^{2} 2\left(1-\cos \ell_{2}\right)}\left[\sin \left(k-\ell_{1}-\ell_{2}\right)-\sin \left(k-\ell_{1}\right)\right. \\
- & \left.\sin \left(k-\ell_{2}\right)+\sin k-\sin \left(k-\ell_{2}\right)+\sin k+\sin \left(k+\ell_{1}-\ell_{2}\right)-\sin \left(k+\ell_{1}\right)\right] \sin \omega k .
\end{aligned}
$$

Proof: The proof of (14) obtained by using (13) and (3).

Example 4.4 Taking $k=\pi, \ell_{1}=\pi$ and $\ell_{2}=\frac{\pi}{2}$, we get

$$
\begin{aligned}
b_{\omega}= & \frac{\ell_{1}+\ell_{2}}{\pi} \sum_{r_{2}=0}^{2}\left(\pi-r_{2} \frac{\pi}{2}\right) \sin \omega\left(\pi-r_{2} \frac{\pi}{2}\right)+\sum_{r_{2}=0}^{0}\left(\pi-r_{2} \frac{\pi}{2}-\pi\right) \\
& \sin \omega\left(\pi-r_{2} \frac{\pi}{2}-\pi\right)-\sum_{r_{2}=0}\left(-r_{2} \frac{\pi}{2}\right) \sin \omega\left(-r_{2} \frac{\pi}{2}\right)=\frac{\ell_{1}+\ell_{2}}{\pi} \pi \sin \omega(\pi)+\frac{\pi}{2}
\end{aligned}
$$

Particularly taking $\eta=3$, we obtain $b_{1}=\frac{\ell_{1}+\ell_{2}}{\pi}, b_{2}=0$ and $k=\frac{l_{1}+l_{2}}{2} \sin k$. 
Theorem 4.5 Let $u(k)=k^{2}, \ell_{1}$ and $\ell_{2}>0$, then GFF $S_{2}$ of $k^{2}$ is

$$
k^{2}=\frac{\ell_{1}+\ell_{2}}{\pi} \sum_{\omega=1}^{\eta} \sum_{r_{1}=0}^{\left[\frac{k}{\ell_{1}}\right]} \sum_{r_{2}=0}^{\left[\frac{k-r_{1} \ell_{1}}{\ell_{2}}\right]}\left(k-r_{2} \ell_{2}-r_{1} \ell_{1}\right)^{2}\left(\frac{1}{2}+\cos \omega\left(k-r_{2} \ell_{2}-r_{1} \ell_{1}\right) \cos \omega k\right) .
$$

Proof: By using (12) and (3), we get the proof of (15).

Theorem 4.6 Let $u(k)=k^{3}, \ell_{1}$ and $\ell_{2}>0$, then $G F F S_{2}$ of $k^{3}$ is

$$
k^{3}=\frac{\ell_{1}+\ell_{2}}{\pi} \sum_{\omega=1}^{\eta-1} \sum_{r_{1}=0}^{\left[\frac{k}{\ell_{1}}\right]} \sum_{r_{2}=0}^{\left[\frac{k-r_{1} \ell_{1}}{\ell_{2}}\right]}\left(k-r_{2} \ell_{2}-r_{1} \ell_{1}\right)^{3} \sin \omega\left(k-r_{2} \ell_{2}-r_{1} \ell_{1}\right) \sin \omega k .
$$

Proof: The proof of (16) obtained by using $(13)$ and $(3)$.

Theorem 4.7 Let $u(k)=k \sin k, \ell_{1}$ and $\ell_{2}>0$, then $G F F S_{2}$ of $k \sin k$ is

$$
k \sin k=\frac{a_{0}}{2}+\sum_{\omega=1}^{\eta-1} a_{\omega} \cos \omega k+\frac{a_{\eta}}{2} \cos \eta k .
$$

Here $a_{\omega}=\left.\frac{\ell_{1}+\ell_{2}}{2 \pi} \Delta_{\ell_{2}}^{-1} \Delta_{\ell_{1}}^{-1} k \sin k \cos \omega\right|_{0} ^{\pi}$

Proof: The proof of (17) obtained by using $(13)$ and (3).

Example 4.8 Taking $\ell_{1}=\frac{\pi}{2}, \ell_{2}=\frac{\pi}{4}$ in 17 , we arrive

$$
\begin{aligned}
a_{\omega}= & \left.\frac{\ell_{1}+\ell_{2}}{2 \pi} \sum_{r_{1}=0}^{\left[\frac{k}{\ell_{1}}\right]} \sum_{r_{2}=0}^{\left[\frac{k-r_{1} \ell_{1}}{\ell_{2}}\right]}\left(k-r_{2} \ell_{2}-r_{1} \ell_{1}\right) \sin \left(k-r_{2} \ell_{2}-r_{1} \ell_{1}\right) \cos \omega\left(k-r_{2} \ell_{2}-r_{1} \ell_{1}\right)\right|_{0} ^{\pi} \\
= & \frac{\ell_{1}+\ell_{2}}{2 \pi}\left(\sum_{r_{2}=0}^{4}\left(\pi-r_{2} \frac{\pi}{4}\right) \sin \left(\pi-r_{2} \frac{\pi}{4}\right) \cos \omega\left(\pi-r_{2} \frac{\pi}{4}\right)+\sum_{r_{2}=0}^{2}\left(\pi-r_{2} \frac{\pi}{4}-\frac{\pi}{2}\right)\right. \\
& \sin \left(\pi-r_{2} \frac{\pi}{4}-\frac{\pi}{2}\right) \cos \omega\left(\pi-r_{2} \frac{\pi}{4}-\frac{\pi}{2}\right)+\sum_{r_{2}=0}\left(\pi-r_{2} \frac{\pi}{4}-\pi\right) \sin \left(\pi-r_{2} \frac{\pi}{4}-\pi\right)
\end{aligned}
$$




$$
\begin{gathered}
\left.\quad \cos \omega\left(\pi-r_{2} \frac{\pi}{4} \pi\right)-\sum_{r_{2}=0}\left(-r_{2} \frac{\pi}{4}\right) \sin \left(-r_{2} \frac{\pi}{4}\right) \cos \omega\left(-r_{2} \frac{\pi}{4}\right)\right) \\
a_{\omega}=\frac{\ell_{1}+\ell_{2}}{2 \pi}\left(0.09686 \cos \omega\left(\frac{3 \pi}{4}\right)+0.043055 \cos \omega\left(\frac{\pi}{2}\right)\right. \\
\left.+0.010765 \cos \omega\left(\frac{\pi}{4}\right)+0.043055 \cos \omega\left(\frac{\pi}{2}\right)\right) .
\end{gathered}
$$

Particularly taking $\eta=3$, we get $k \sin k=\frac{\ell_{1}+\ell_{2}}{4 \pi}(0.193735)+\frac{\ell_{1}+\ell_{2}}{2 \pi}(0.193608) \cos k$ $+\frac{\ell_{1}+\ell_{2}}{2 \pi}(0.193269) \cos 2 k+\frac{\ell_{1}+\ell_{2}}{4 \pi}(0.192697) \cos 3 k$.

\section{Conclusion}

The Fourier series and its transforms have wide range of applications specially in the field of digital signal processing. For the odd and even functions no usual finite half range Fourier series expression, we are able to find finite Fourier series expression (decomposition) using generalized inverse difference operator with two parameters.

\section{References}

[1] Anguelov.R, Spline-Fourier approximation of discontinuous waves, J. Univ. Comput. Sci. 4(2) (1998).

[2] Britto Antony Xavier.G, Sathya.S, Vasanthakumar.S.U, m-Series of the Generalized Difference Equation to Circular Functions,International Journal of Mathematical Archive-4(7), 200-209 (2013).

[3] Edwards R F, Fourier Series, A Modern Introduction, Vol.1. Holt, Rinehart and Winston, New York, 1967.

[4] Govindan.B, Nandhini.M, Meganathan.M, Generalized Discrete Finite Half Range Fourier Series,Advanced in Mathematics: Scientific Journal, 9, 1225-1237 (2020).

[5] Jerri.A.J, The Gibbs Phenomenon in Fourier Analysis, Splines and Wavelet Approximations, Kluwer Academic Publishers, Dordrecht-Bosten-London, 1998.

[6] Maria Susai Manuel.M, Britto Antony Xavier.G and Thandapani.E, Theory of Generalized Difference Operator and Its Applications, Far East Journal of Mathematical Sciences, 20(2), 163 - 171 (2006). 
ISSN: 2456-8686, 5(1), 2021:088-096

https://doi.org/10.26524/cm95

[7] Sidi.A, Practical Extrapolation Methods: Theory and Applications, Cambridge University Press, 2005.

[8] Steven W. Smith, The Scientist and Engineer's Guide to Digital Signal Processing, Second Edition, California Technical Publishing San Diego, California, 1999.

[9] Tolstov.G.P, Fourier series, Dover, New York, 1976. 